\title{
Lithium related deep and shallow acceptors in Li-doped ZnO nanocrystals
}

Cite as: J. Appl. Phys. 107, 024311 (2010); https://doi.org/10.1063/1.3275889

Submitted: 31 August 2009 . Accepted: 23 November 2009 . Published Online: 27 January 2010

C. Rauch, W. Gehlhoff, M. R. Wagner, E. Malguth, G. Callsen, R. Kirste, B. Salameh, A. Hoffmann, S. Polarz, Y. Aksu, and M. Driess

\section{ARTICLES YOU MAY BE INTERESTED IN}

A comprehensive review of $\mathrm{ZnO}$ materials and devices

Journal of Applied Physics 98, 041301 (2005); https://doi.org/10.1063/1.1992666

Control of $p$ - and $n$-type conductivities in Li-doped ZnO thin films

Applied Physics Letters 89, 112113 (2006); https://doi.org/10.1063/1.2354034

Oxygen vacancies in $\mathrm{ZnO}$

Applied Physics Letters 87, 122102 (2005); https://doi.org/10.1063/1.2053360

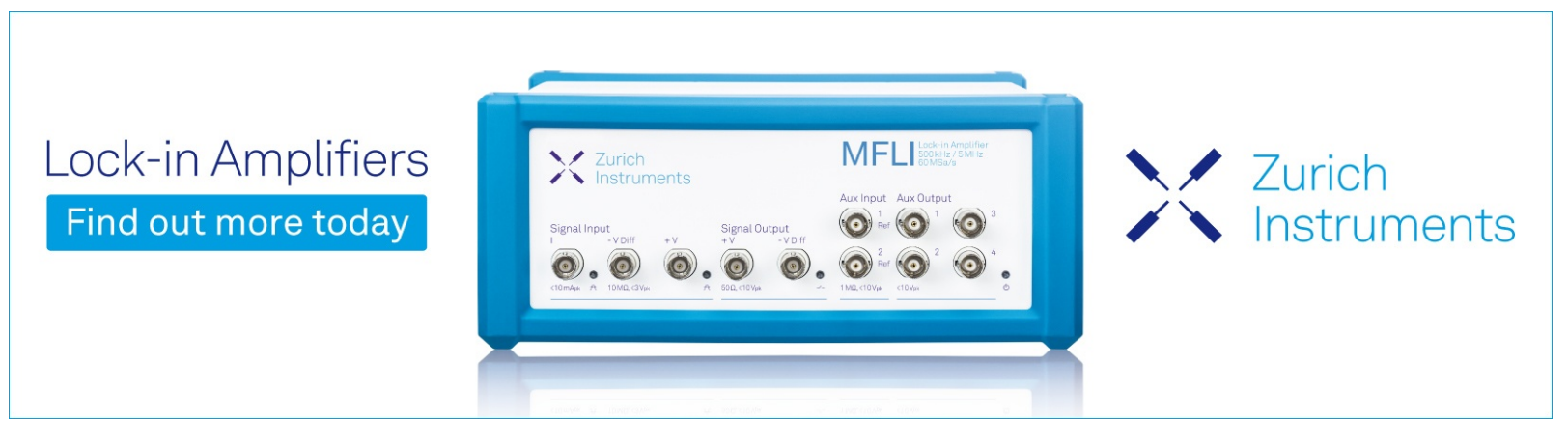




\title{
Lithium related deep and shallow acceptors in Li-doped ZnO nanocrystals
}

\author{
C. Rauch, ${ }^{1, a)}$ W. Gehlhoff, ${ }^{1}$ M. R. Wagner, ${ }^{1}$ E. Malguth, ${ }^{1}$ G. Callsen, ${ }^{1}$ R. Kirste,${ }^{1}$ \\ B. Salameh, ${ }^{1,2}$ A. Hoffmann, ${ }^{1}$ S. Polarz, ${ }^{3}$ Y. Aksu, ${ }^{4}$ and M. Driess ${ }^{4}$ \\ ${ }_{1}^{1}$ Institute of Solid State Physics, TU Berlin, Hardenbergstrasse 36, D-10623 Berlin, Germany \\ ${ }^{2}$ Department of Applied Physics, Tafila Technical University, Tafila 66110, Jordan \\ ${ }^{3}$ Department of Chemistry, University of Konstanz, D-78457 Konstanz, Germany \\ ${ }^{4}$ Institute of Chemistry, Metalorganics and Inorganic Materials, TU Berlin, Straße des 17. Juni 135, \\ D-10623 Berlin, Germany
}

(Received 31 August 2009; accepted 23 November 2009; published online 27 January 2010)

\begin{abstract}
We study the existence of Li-related shallow and deep acceptor levels in Li-doped $\mathrm{ZnO}$ nanocrystals using electron paramagnetic resonance (EPR) and photoluminescence (PL) spectroscopy. ZnO nanocrystals with adjustable $\mathrm{Li}$ concentrations between $0 \%$ and $12 \%$ have been prepared using organometallic precursors and show a significant lowering of the Fermi energy upon doping. The deep $\mathrm{Li}$ acceptor with an acceptor energy of $800 \mathrm{meV}$ could be identified in both EPR and PL measurements and is responsible for the yellow luminescence at $2.2 \mathrm{eV}$. Additionally, a shallow acceptor state at $150 \mathrm{meV}$ above the valence band maximum is made responsible for the observed donor-acceptor pair and free electron-acceptor transitions at 3.235 and $3.301 \mathrm{eV}$, possibly stemming from the formation of Li-related defect complexes acting as acceptors. (C) 2010 American Institute of Physics. [doi:10.1063/1.3275889]
\end{abstract}

$\mathrm{ZnO}$ is a II-VI compound semiconductor with a direct band gap of $3.437 \mathrm{eV}^{1}$ at $T=1.6 \mathrm{~K}$. It regained substantial interest over the past decade, which has been mainly fueled by considerable progress in crystal growth that resulted in the fabrication of high quality crystal samples in various structures. ${ }^{2}$ One of the key features of $\mathrm{ZnO}$ is its high exciton binding energy of $E_{B}=60 \mathrm{meV}$ that, e.g., gives hope for the realization of high efficient lasing at room temperature. $\mathrm{ZnO}$ is known for its strong tendency to self-organization and the preparation of a variety of different $\mathrm{ZnO}$ based nanostructures with a multitude of interesting applications was reported in the last years. ${ }^{2}$ The key challenge that needs to be overcome for the realization of most $\mathrm{ZnO}$ based applications is the fabrication of p-type material. P-type $\mathrm{ZnO}$ may be achieved by the substitution of group-I elements on $\mathrm{Zn}$-site and group- $\mathrm{V}$ elements on $\mathrm{O}$-site, respectively. Although significant progress has been made recently ${ }^{3}$ full control over the materials conductivity type is still to be obtained and hence a comprehensive investigation of the fundamental properties of acceptors in $\mathrm{ZnO}$ is needed.

The interest in $\mathrm{Li}$ as a dopant in $\mathrm{ZnO}$ is based on both its possible ability to act as a p-dopant in $\mathrm{ZnO}$, as well as on the report of ferroelectricity in $\mathrm{ZnO}$ upon Li-doping. ${ }^{4}$ Additionally, $\mathrm{Li}$ is known to act as a major impurity in $\mathrm{ZnO}$ growth. Theoretical calculations have predicted that substitutional $\mathrm{Li}$ on $\mathrm{Zn}$-site $\left(\mathrm{Li}_{\mathrm{Zn}}\right)$ has the lowest acceptor energy of single impurity dopants in $\mathrm{ZnO}$ with $90 \mathrm{meV}$ above the valence band maximum (VBM). ${ }^{5}$ However, until recently only a deep acceptor at around $800 \mathrm{meV}$ above the VBM has been observed commonly in Li-doped $\mathrm{ZnO}$ structures. Due to its small ionic radius $\mathrm{Li}$ is additionally known to be very mobile

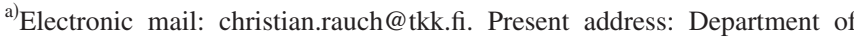
Applied Physics, Helsinki University of Technology, P.O. Box 1100, FI02015 TKK, Finland.
}

in the $\mathrm{ZnO}$-lattice and can easily occupy interstitial positions $\left(\mathrm{Li}_{i}\right)$, where it acts as donor. This amphoteric behavior, together with the formation of the deep $\mathrm{Li}$ acceptor trap, has been made responsible for the frequent observation of semiinsulating material upon $\mathrm{Li}$ doping of $\mathrm{ZnO}$ and accentuates the significance of a controlled incorporation of $\mathrm{Li}$ dopants on the Zn-lattice site. ${ }^{6}$ Nevertheless, there have been recent reports about the observation of shallow $\mathrm{Li}$ acceptors ${ }^{7-9}$ which indicate the great potential of $\mathrm{Li}$ as a p-dopant in $\mathrm{ZnO}$. However, a comprehensive understanding of the role of $\mathrm{Li}$ as dopant in $\mathrm{ZnO}$ has not been established so far. In this contribution, we use electron paramagnetic resonance (EPR) and photoluminescence (PL) spectroscopy to prove the coexistence of a deep and shallow acceptor state in nanocrystalline Li-doped $\mathrm{ZnO}$, showing a significantly lower Fermi energy.

Li-doped $\mathrm{ZnO}$ nanocrystals with adjustable Li concentration from $0 \%$ up to $12 \%$ have been prepared..$^{10}$ The given $\mathrm{Li}$ concentrations correspond here to the values determined by the atomic ratios of the mixture of two precursor systems. A detailed description of the synthesis of $\mathrm{Li}$-containing $\mathrm{ZnO}$ materials from special organometallic precursors ${ }^{11}$ is beyond the scope of this article and can be found elsewhere. ${ }^{10} \mathrm{EPR}$ measurements were carried out in the Q-band $(35 \mathrm{GHz})$ at temperatures between 4 and $300 \mathrm{~K}$ using a Bruker ESP 300E spectrometer equipped with an Oxford Instrument continuous-flow cryostat. The samples could be optically excited by the $325 \mathrm{~nm}$ and/or $442 \mathrm{~nm}$ lines of a He-Cd laser through an optical fiber introduced in the Q-band cryostat. PL measurements were performed using the $325 \mathrm{~nm}$ line of a $\mathrm{He}-\mathrm{Cd}$ laser. The emission was dispersed by a $0.8 \mathrm{~m}$ Spex double monochromator with a resolution better than $0.1 \mathrm{meV}$ and detected by a bialkali photomultiplier. For time-resolved PL (TRPL) measurements the samples were excited using the second harmonic of a titan sapphire laser $(3.480 \mathrm{eV})$ with a pulse length of $2 \mathrm{ps}$. Time-resolved data were recorded by 
single photon counting using a Hamamatsu R3809U-52 microchannel plate. The instrumental time resolution is about $40 \mathrm{ps}$ which allows the determination of lifetimes down to 15 ps using deconvolution techniques.

The incorporation of $\mathrm{Li}$ and related defects into the $\mathrm{Li}$ doped samples, as well as the undoped reference sample, was examined by EPR measurements. Without additional illumination only the signal from the shallow donor and the very weak signals from $\mathrm{Mn}^{2+}$ were observed in the undoped reference sample. In the Li-doped samples the EPR signals of $\mathrm{Mn}^{2+}$ and $\mathrm{Fe}^{3+}$ on $\mathrm{Zn}^{2+}$ site but no signal from the shallow donor at $g=1.956^{1,12}$ could be detected. In order to be able to monitor the presence of ionized $\mathrm{Li}^{+}$acceptors the defect has to be reloaded to a paramagnetic state ${ }^{13}$ by optical irradiation of the material. After illumination with a $\mathrm{He}-\mathrm{Cd}$ laser at 325 $\mathrm{nm}$ and/or $442 \mathrm{~nm}$ at temperatures below $200 \mathrm{~K}$ the shallow donor signal, the weak signal probably from the negatively charged zinc vacancy $V_{\mathrm{Zn}},{ }^{12,14}$ and also the characteristic spectrum of the deep $\mathrm{Li}$ acceptor center $\mathrm{Li}_{\mathrm{Zn}}$ were detected in the Li-doped samples.

Detailed EPR investigations of the $\mathrm{Li}$ acceptor in $\mathrm{Li}$ doped $\mathrm{ZnO}$ single crystals by Schirmer ${ }^{13}$ have shown that the $\mathrm{Li}$ spectrum stems from $\mathrm{Li}^{+}-\mathrm{O}^{-}$centers in the material, which are formed by the capture of a hole at one of the four $\mathrm{O}^{2-}$ ions surrounding the substitutional $\mathrm{Li}^{+}$ion during optical irradiation. The spectra of the axial and nonaxial $\mathrm{Li}^{+}-\mathrm{O}^{-}$ defects can be described by the spin Hamiltonian $(\mathrm{SH})$

$$
\mathcal{H}=\mu_{B} \mathrm{SgB}+\mathrm{IAS}+\mathrm{IPI}-g_{N} \mu_{N} \mathrm{BI} .
$$

with $\mathrm{S}=1 / 2$ and $\mathrm{I}=3 / 2$ for ${ }^{7} \mathrm{Li}$ (natural abundance of $92.41 \%$ ) and $\mathrm{I}=1$ for ${ }^{6} \mathrm{Li}$ (natural abundance of $7.59 \%$ ). The used parameters have their conventional meaning, whose magnitudes and principal axes have been determined by Schirmer. ${ }^{13}$ However, these four positions are not equivalent due to the polar nature of hexagonal $\mathrm{ZnO}$. For substitutional Li the axial configuration with the hole trapped at an oxygen ion found along the c-axis is favored over the three nonaxial hole positions. The energy separation between the axial and the three nonaxial hole positions (center orientations) has been determined to $15 \mathrm{meV}$ by the variation in the equilibrium population of both types of sites with the temperature. ${ }^{13}$ For the calculation of the corresponding $\mathrm{Li}$ spectrum in $\mathrm{ZnO}$ powder, a random distribution of the orientation of the axes of the nanocrystals has to be taken into account. This was done by integrating the EPR line positions of the different center orientations over the full space angle. Using the EASYSPIN software package ${ }^{15}$ and the $\mathrm{SH}$ parameters determined by Schirmer ${ }^{13}$ we solved the SH (Eq. (1)) by exact diagonalization for the axial and the nonaxial $\mathrm{Li}^{+}-\mathrm{O}^{-}$center, while taking into consideration that there are two cation sites in the unit cell of a hexagonal crystal.

The resulting Q-band EPR spectra are shown for the axial and nonaxial defects in Figs. 1(a) and 1(b), respectively. The experimentally observed spectrum is theoretically described by the weighted sum of both spectra, which is shown for different intensities of the two parts of the total spectrum in Figs. 1(c) and 1(d), respectively. The spectrum in Fig. 1(d) results from considering the ratio of axial to nonaxial lattice sites of $1: 3(2: 6)$, assuming that the capture

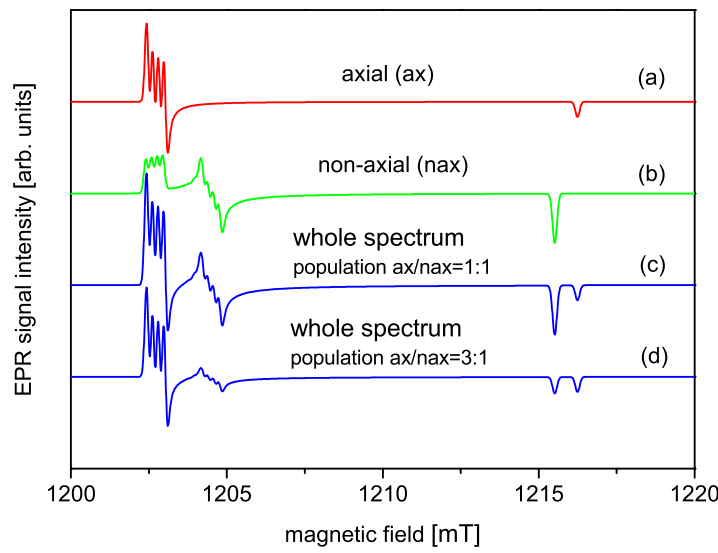

FIG. 1. (Color online) Calculated EPR spectra of the (a) axial and (b) nonaxial defects in an ensemble of $\mathrm{ZnO}$ nanocrystals using the $\mathrm{SH}$ parameters given by Schirmer for single crystals ${ }^{13}$ and the resulting sum spectra $[(\mathrm{c})$ and (d)] for two different population ratios of the axial and nonaxial defects.

of a hole at the four surrounding oxygen ions occurs with equal probability. Additionally, for comparing these theoretical spectra with our experimentally obtained values, one has to consider that due to the illumination of the samples there is a competition between the trapping of the holes and the temperature dependent depopulation of the nonaxial defects. Therefore, the resulting relative intensity between both spectra is dependent on the arrived steady-state population of the two types of defects that depends on the temperature and on the intensity of the laser.

A comparison of the calculated and experimentally obtained Q-band EPR spectra of the Li acceptor centers under laser excitation at $442 \mathrm{~nm}$ and $T=30 \mathrm{~K}$ is displayed in Fig. 2 exemplarily for nanocrystals with a nominal $\mathrm{Li}$ concentration of $6 \%$. In order to resolve the ${ }^{7} \mathrm{Li}$ hyperfine splitting a diminutive modulation amplitude of $0.06 \mathrm{mT}$ was used and the spectra are restricted to the small magnetic field region relevant for the $\mathrm{Li}$ acceptor resonance. Concerning the line positions and the ${ }^{7} \mathrm{Li}$ hyperfine splitting of both the axial and

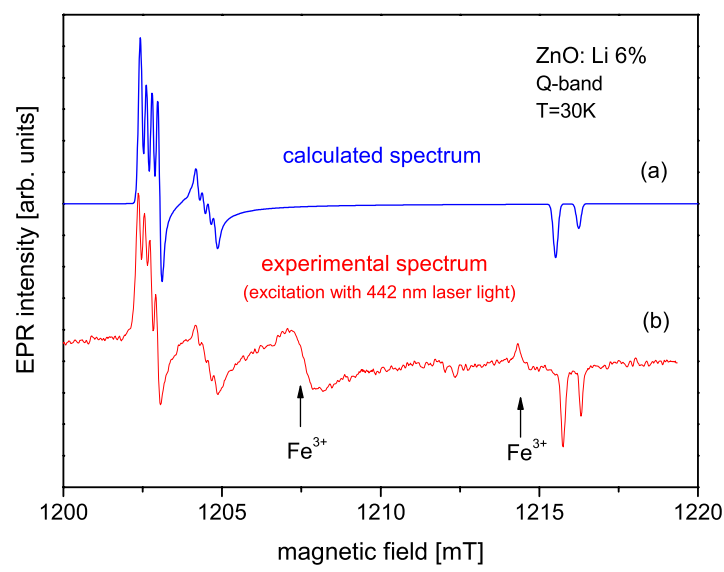

FIG. 2. (Color online) Comparison of calculated and experimental EPR spectra of the $\mathrm{Li}^{+}-\mathrm{O}^{-}$defect for nanocrystalline $\mathrm{ZnO}$ in the Q-band (34 $\mathrm{GHz}$ ) at $T=30 \mathrm{~K}$. (a) Calculated spectrum for the axial and nonaxial $\mathrm{Li}^{+}-\mathrm{O}^{-}$defects for a population ratio of 1:2. (b) Experimental spectrum of a $\mathrm{ZnO}$ nanocrystals ensemble with nominal $6 \% \mathrm{Li}$ under illumination with laser light of $442 \mathrm{~nm}$. Besides the $\mathrm{Li}$ spectrum two $\mathrm{Fe}_{\mathrm{Zn}}^{3+}$ lines, which are indicated by arrows, are observed in the shown magnetic field region. 
nonaxial $\mathrm{Li}^{+}-\mathrm{O}^{-}$defects, excellent agreement is found between the experimental spectrum and the simulated one. This confirms that the observed EPR spectrum in the nanocrystalline $\mathrm{ZnO}$ samples originates from the same $\mathrm{Li}^{+}-\mathrm{O}^{-}$defect as found in single crystals. Similar observations for Li concentrations of $1 \%$ and $12 \%$ demonstrate the successful incorporation of $\mathrm{Li}_{\mathrm{Zn}}$ in all our samples. It is well established that this $\mathrm{Li}$ acceptor participates in a broad luminescence band centered at around $2.2 \mathrm{eV}$ that can be explained by a shallow donor-deep acceptor recombination, which could also be observed in our PL measurements upon Li doping of the nanocrystals (not displayed here). From the edge of the luminescence band at around $2.5 \mathrm{eV}$, an acceptor energy of $0.8 \mathrm{eV}$ above the VBM for $\mathrm{Li}_{\mathrm{Zn}}$ is estimated. ${ }^{16}$

Besides this Li acceptor, additional unintentionally introduced defects could be detected in the EPR spectra. As mentioned above, we observed the signals of $\mathrm{Mn}^{2+}$ and $\mathrm{Fe}^{3+}$ in all Li-doped samples in measurements without illumination. The two $\mathrm{Fe}^{3+}$ lines occurring in the magnetic field region between 1200 and $1220 \mathrm{mT}$ are indicated by arrows in Fig. 2. The signals of $\mathrm{Mn}^{2+}$ are not visible here because they are suppressed by saturation under the selected experimental conditions to optimize the $\mathrm{Li}$ resonance. Under optical irradiation to produce the $\mathrm{Li}$ resonance, in some samples additional weak signals probably caused by the $V_{\mathrm{Zn}}^{-}$, became visible. This spectrum is investigated in detail for electron irradiated $\mathrm{ZnO}$ single crystals. ${ }^{12,14}$ In our nanocrystals, these weak signals are difficult to detect because they are severely overlapped with the strong low-field $\mathrm{Fe}^{3+}$ line and the highfield line of the nonaxial Li centers. More important for the characterization of the investigated nanocrystals is the observation of $\mathrm{Fe}^{3+}$ spectrum in the "dark" measurements. The fact that in contrast to the undoped reference sample, Fe can be detected in its $3+$ charge state in all the Li-doped samples even without additional illumination determines that the Fermi energy is pushed down at or below the $\mathrm{Fe}^{2+} / \mathrm{Fe}^{3+}$ charge transfer level due to the Li-doping. This defines the Fermi energy in the Li-doped samples to be located below around $2.5 \mathrm{eV}$ above the VBM. ${ }^{17,18}$ The presence of $\mathrm{Fe}^{3+}$ in the Li-doped samples could additionally be verified by high resolution PL measurements of the deep center emission of the samples, for which the distinct complex $\mathrm{Fe}^{3+}$ emission feature was detected for all investigated Li concentrations.

It has been suggested that besides the deep $\mathrm{Li}_{\mathrm{Zn}}$ center $\mathrm{Li}$ might also form shallow acceptor levels, e.g., through the formation of defect complexes. ${ }^{7}$ These complexes could lower the strong distortion in the $\mathrm{Li}$ center which has been made responsible for the high binding energy. ${ }^{13}$ Figure 3 shows the near band edge PL spectra of the samples with $0 \%, 1 \%, 6 \%$, and $12 \% \mathrm{Li}$ at $T=2 \mathrm{~K}$. The dominant luminescence channels in the near band gap area of our samples are the bound exciton emission (BX) at $3.360 \mathrm{eV}$, centered around the neutral donor bound exciton line $\mathrm{I}_{8},{ }^{1}$ together with a strong luminescence at $3.315 \mathrm{eV}$ (SX), which has been observed previously in $\mathrm{ZnO}$ nanocrystals and was indeed attributed to excitons bound to acceptors. Nevertheless, these acceptor states are caused by structural defects near the surface of the crystals ${ }^{19}$ rather than intentionally introduced dopants. Schirra et al. ${ }^{20}$ proved in a recent comprehensive

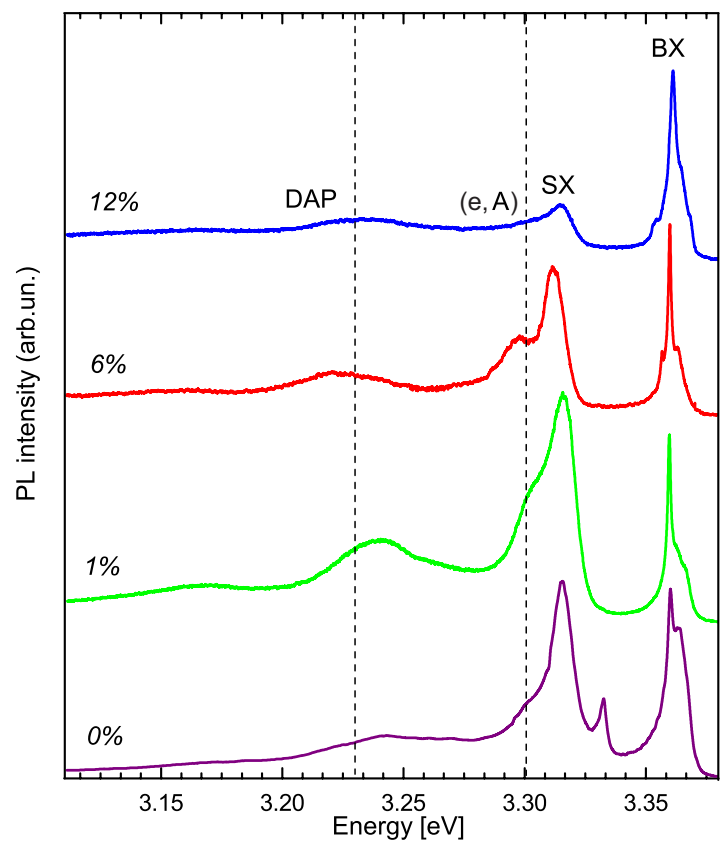

FIG. 3. (Color online) PL spectra taken at $T=2 \mathrm{~K}$ of ensembles of Lidoped $\mathrm{ZnO}$ nanocrystals with $\mathrm{Li}$ concentrations of $0 \%, 1 \%, 6 \%$, and $12 \%$. All spectra are normalized to the $\mathrm{BX}$ at $3.36 \mathrm{eV}$.

study that the observation of the $3.31 \mathrm{eV}$ luminescence in various $\mathrm{ZnO}$ structures stems from a common origin which can be assigned to basal plane stacking faults.

More interesting concerning the question of a possible shallow $\mathrm{Li}$ acceptor is the luminescence line located at an energy of $3.235 \mathrm{eV}$ that becomes visible upon Li doping of the nanocrystals. Furthermore a closer look at the luminescence around $3.31 \mathrm{eV}$ reveals a shoulder at $3.301 \mathrm{eV}$ in our samples, which seems to increase with the Li concentration. In the following we will assign these recombinations to a donor-acceptor pair (DAP) and corresponding free electron to acceptor (e,A) transition, involving the same acceptor state. The luminescence at $3.235 \mathrm{eV}$ is most prominent at a $\mathrm{Li}$ concentration of $1 \%$ and decreases with increasing Li content. XRD and Raman measurements of our samples demonstrated the highest sample quality and lowest strain for the $1 \% \mathrm{Li}$ sample. ${ }^{10} \mathrm{Lu}$ et al. ${ }^{21}$ reported the largest free hole concentration in $\mathrm{Li}$-doped $\mathrm{ZnO}$ for a $\mathrm{Li}$ content of 0.6 at. \%, whereas higher Li concentrations result in compensated material. This compensation is expressed in our PL spectra by a decrease in intensity of the $3.235 \mathrm{eV}$ luminescence as well as a red-shift with increasing Li concentration. A pronounced phonon coupling of the luminescence can be observed. The broadened lineshape compared to the exciton luminescence and the shift to lower energies for higher dopant concentrations is well known for DAP recombinations in compensated material. ${ }^{22}$

In order to support the assignment of the $3.235 \mathrm{eV}$ luminescence to a DAP recombination, TRPL measurements were performed. Figure 4 displays the transients at the BX, $\mathrm{SX}$, and DAP peak energies in the 1\% Li-doped sample. Evidently, all three transitions demonstrate very different recombination dynamics. The BX line at $3.360 \mathrm{eV}$ exhibits primarily a monoexponential decay characteristic with a time 


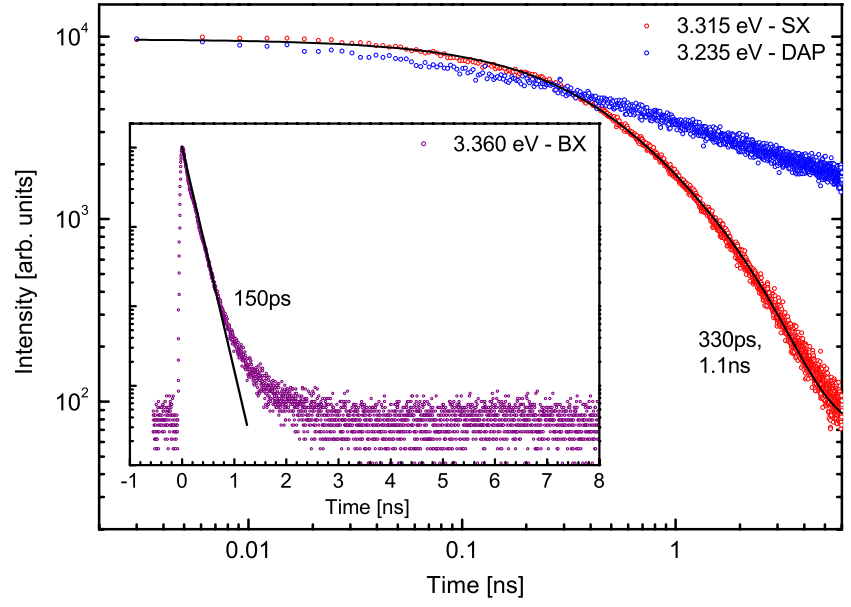

FIG. 4. (Color online) TRPL of the $3.360 \mathrm{eV}$ (BX), $3.315 \mathrm{eV}$ (SX), and $3.235 \mathrm{eV}$ (DAP) transitions in $1 \%$ Li-doped $\mathrm{ZnO}$ nanocrystals.

constant of 150 ps which is typical for bound exciton recombinations in $\mathrm{ZnO}$ nanostructures. ${ }^{23}$ The recombination dynamics of the SX peak at $3.315 \mathrm{eV}$ is governed by a biexponential decay with lifetimes of $330 \mathrm{ps}$ and $1.1 \mathrm{~ns}$ in agreement with values reported by Fallert et al. ${ }^{19}$ By contrast, the decay dynamics of the $3.235 \mathrm{eV}$ luminescence shows a broad distribution of lifetimes that extends beyond the time window of $12.5 \mathrm{~ns}$ between two laser pulses and cannot be described by a mono or biexponential decay. This behavior is expected for a DAP recombination due to the varying separations of shallow donors and shallow acceptors. ${ }^{24}$ Consequently, we attribute this transition to a DAP recombination. The large difference in the decay behavior of the 3.315 and $3.235 \mathrm{eV}$ further excludes the possibility of a one-LO phonon replica as the origin of the $3.235 \mathrm{eV}$ luminescence. The binding energy of the acceptor involved in the DAP transition is estimated by

$$
E_{A}^{b}=E_{G}-E_{\mathrm{DAP}}-E_{D}^{b}+\frac{e^{2}}{4 \pi \varepsilon \varepsilon_{0} r_{D A}}, \cong 150 \mathrm{meV},
$$

with $r_{D A}$ being the average distance between the donor and acceptor defects and $E_{D}^{b}=54.6 \mathrm{meV}$ the binding of the dominant $\mathrm{I}_{8}$ donor. ${ }^{1}$ Remarkably, this acceptor energy corresponds well to the energy of the luminescence at $3.301 \mathrm{eV}$, which additionally shows a constant intensity ratio with respect to the $3.235 \mathrm{eV}$ luminescence in the samples with $1 \%$ and $6 \%$ Li. In the $12 \%$ sample both luminescences are strongly suppressed, indicating poor sample quality and high compensation.

Based on these observations we suggest following explanation of the luminescence spectra of the Li-doped $\mathrm{ZnO}$ nanocrystals. Besides the deep $\mathrm{Li}_{\mathrm{Zn}}$ acceptor at $0.8 \mathrm{eV}$ above the VBM, which is responsible for the observed yellow luminescence, the Li-doping of the $\mathrm{ZnO}$ nanocrystals leads to the formation of an additional shallow acceptor, possibly through the formation of a defect complex. This shallow acceptor participates in a DAP luminescence at $3.235 \mathrm{eV}$ as well as a $(\mathrm{e}, \mathrm{A})$ transition at $3.301 \mathrm{eV}$ and possesses an energy level located around $150 \mathrm{meV}$ above the VBM. Recently, comparable shallow acceptor energies have also been reported in reactive magnetron sputtered $\mathrm{Li}$-doped $\mathrm{ZnO}$ films ${ }^{8}$ and $\mathrm{Li}-\mathrm{F}$ codoped $\mathrm{ZnO} .^{9}$ This suggests that through controlled introduction of Li-defect complexes p-type doping of $\mathrm{ZnO}$ could be achieved. Further investigations are called for to clarify the chemical origin as well the formation mechanisms of shallow Li acceptors.

In summary, we presented the identification of shallow and deep acceptors in Li-doped $\mathrm{ZnO}$ nanocrystals. $\mathrm{ZnO}$ nanocrystals with $\mathrm{Li}$ concentrations between $0 \%$ and $12 \%$ were grown using organometallic precursors and show a significant lowering of the Fermi level compared to undoped material. A detailed analysis with EPR showed strong contributions of the deep $\mathrm{Li}_{\mathrm{Zn}}$ which has been made responsible for the yellow luminescence in $\mathrm{ZnO}$ hence possessing an acceptor energy of around $800 \mathrm{meV}$. Besides the deep Li acceptor, PL measurements also suggest the existence of a shallow acceptor at $150 \mathrm{meV}$ above the VBM in the Lidoped samples, which is visible in the PL spectrum from a DAP luminescence at $3.235 \mathrm{eV}$ and a $(\mathrm{e}, \mathrm{A})$ transition at $3.301 \mathrm{eV}$. Tentatively, a Li-related defect complex is made responsible for this additional shallow acceptor state. The incorporation of this shallow $\mathrm{Li}$ acceptor seems to be most efficient for a Li concentration around 1\%. Higher doping leads to compensated material with lower structural quality.

We thank the Grillo-group and Grillo Zinkoxid GmbH for financial support. The Deutsche Forschungsgemeinschaft (DFG) is acknowledged for funding (SFB 787; Project Nos. PO780/4-1 and DR 226/16-1). A. Orlov is acknowledged for assistance in the synthesis of the Li-containing $\mathrm{ZnO}$ materials. M.R.W. and C.R. acknowledge an Ernst-von-Siemens scholarship and a Marie Curie ITN fellowship, respectively. B.S. acknowledges an Erasmus Mundus External Cooperation scholarship and support by the DFG (Project No. GE 709/11-1).

${ }^{1}$ B. K. Meyer, H. Alves, D. M. Hofmann, W. Kriegseis, D. Forster, F. Bertram, J. Christen, A. Hoffmann, M. Strassburg, M. Dworzak, U. Haboeck, and A. V. Rodina, Phys. Status Solidi B 241, 231 (2004).

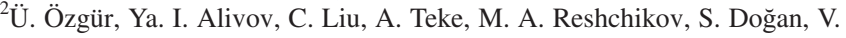
Avrutin, S.-J. Cho, and H. Morkoç, J. Appl. Phys. 98, 041301 (2005).

${ }^{3}$ D. C. Look and B. Claflin, Phys. Status Solidi B 241, 624 (2004).

${ }^{4}$ M. G. Wardle, J. P. Goss, and P. R. Briddon, Phys. Rev. B 71, 155205 (2005)

${ }^{5}$ C. H. Park, S. B. Zhang, and S. H. Wei, Phys. Rev. B 66, 073202 (2002).

${ }^{6}$ E. -C. Lee and K.J. Change, Phys. Rev. B 70, 115210 (2004).

${ }^{7}$ J. Sann, A. Hofstaetter, D. Pfisterer, J. Stehr, and B. K. Meyer, Phys. Status Solidi C 3, 952 (2006).

${ }^{8}$ Y. I. Zeng, Z. Z. Ye, G. J. Lu, W. Z. Xu, L. P. Zhu, and B. H. Zhao, Appl. Phys. Lett. 89, 042106 (2006).

${ }^{9}$ K. Kobayashi, Y. Tomita, Y. Maeda, and H. Haneda, Phys. Status Solidi C 5, $3122(2008)$.

${ }^{10}$ S. Polarz, A. Orlov, A. Hoffmann, M. R. Wagner, C. Rauch, R. Kirste, W. Gehlhoff, Y. Aksu, M. Driess, M. W. E. van den Berg, and M. Lehmann, Chem. Mater. 21, 3889 (2009).

${ }^{11}$ S. Jana, Y. Aksu, and M. Driess, Dalton Trans. 2009, 1516.

${ }^{12}$ L. Vlasenko and G. Watkins, Phys. Rev. B 72, 035203 (2005).

${ }^{13}$ O. F. Schirmer, J. Phys. Chem. Solids 29, 1407 (1968).

${ }^{14}$ D. Galland and A. Herve, Phys. Lett. 33A, 1 (1970).

${ }^{15}$ S. Stoll and A. Schweiger, J. Magn. Reson. 178, 42 (2006).

${ }^{16}$ O. F. Schirmer and D. Zwingel, Solid State Commun. 8, 1559 (1970).

${ }^{17}$ R. Heitz, A. Hoffmann, and I. Broser, Phys. Rev. B 45, 8977 (1992).

${ }^{18}$ E. Malguth, A. Hoffmann, and M. R. Phillips, Phys. Status Solidi B 245, 455 (2008)

${ }^{19}$ J. Fallert, R. Hausschild, F. Stelzl, A. Urban, M. Wissinger, H. Zhou, C. Klingshirn, and H. Kalt, J. Appl. Phys. 101, 073506 (2007).

${ }^{20}$ M. Schirra, L. Schneider, A. Reiser, G. M. Prinz, M. Feneberg, J. Bisku- 
pek, U. Kaiser, C. E. Krill, K. Thonke, and R. Sauer, Phys. Rev. B 77, 125215 (2008).

${ }^{21}$ J. G. Lu, Y. Z. Zhang, Z. Z. Ye, Y. J. Zeng, H. P. He, L. P. Zhu, J. Y. Huang, L. Wang, J. Yuan, B. H. Zhao, and X. H. Li, Appl. Phys. Lett. 89 112113 (2006).

${ }^{22}$ M. Behringer, P. Baeume, J. Gutowski, and D. Hommel, Phys. Rev. B 57,
12869 (1998).

${ }^{23}$ L. Schneider, S. V. Zaitsev, G. Bacher, W. Jin, and M. Winterer, J. Appl. Phys. 102, 023524 (2007).

${ }^{24}$ A. Zeuner, H. Alves, D. M. Hofmann, B. K. Meyer, A. Hoffmann, U. Haboeck, M. Strassburg, and M. Dworzak, Phys. Status Solidi B 234, R7 (2002). 